\title{
Systemic lupus Erythematous as an Unusual Cause of Intussusception in Sudanese Woman: Case report
}

\author{
Salwa Dafa Allah Salih Mohammadeen ${ }^{1}$, Amar F.Eldow ${ }^{2}$, Rania Eisa Abdelmutalib ${ }^{1}$, Sara \\ galal osman hamza ${ }^{1}$, Elnour Mohammed Elagib ${ }^{1}$, huyam H.Awadalla ${ }^{1}$, Abubkar Hassan ${ }^{3}$, \\ Abdelkareem A.Ahmed ${ }^{4}$, and Mohammed Elmujtba Adam Essa ${ }^{5}$ \\ ${ }^{1}$ Sudan Medical Specialization Board \\ ${ }^{2}$ Wayne State University School of Medicine \\ ${ }^{3} \mathrm{MCR}$ \\ ${ }^{4} \mathrm{MCRI}$ \\ ${ }^{5}$ Al Fashir University Faculty of Medicine
}

September 25, 2021

\begin{abstract}
A middle-aged Sudanese woman has been presented complained about multiple joint pain, skin rash, chest pain, hair loss, severe abdominal pain associated with abdominal distension, bloody diarrhoea and vomiting. Lab investigation and computed tomography (CT) abdomen revealed the patient have an intussusception on top of SLE. The patient was treated
\end{abstract}

\section{Introduction}

SLE is a complex autoimmune chronic inflammatory disorder with an extensive cascade of symptoms, the disease can involve the kidney, lung, nervous system and heart (1). Abdominal pain does have a wide differential diagnosis, however, gastrointestinal involvement is not unusual in SLE $(2,3)$. A lot of causes of abdominal pain have been described in lupus patients such as lupus enteritis drug reactions and infections due to steroids $(4,5)$. In addition, the likelihood of SLE as a contributing factor in jejunal diverticulosis has been reported (6).

An intussusception itself as a complication or presenting symptom of SLE is rare, until now there are no more than seven cases have been reported in $\operatorname{SLE}(4,6)$. Two of those cases it was secondary to mesenteric vacuities and five cases had an intussusception as an initial presentation of SLE (7). In this case, we are reporting a case of SLE with intussusception as the initial presentation.

\section{Case report}

A 40-year old Sudanese female, presented with complaints of facial skin rash, bilateral small \& large joints pain, swelling, Palpitations, Shortness of breath, Pleuritic chest pain, Hair loss and Muscle pain.

Soon after she has been admitted to our rheumatology unite at Omdurman military hospital, Khartoum Sudan, the patient developed acute severe cramping periumbilical abdominal pain associated with abdominal distension, vomiting \& infrequent bloody diarrhoea. Her examination showed high blood pressure (150/100), pulse rate of 100 , temperature 37.5 , she has malar rash sparing the nasolabial folds with hyperpigmented patches, no rash in other sites, broken hair in the frontal and temporal areas and no nail changed. Cardiovascular examination revealed short localized Systolic murmur in the mitral area, chest examination indicated 
decreased air entry bilaterally with dull percussion notes, her abdomen was distended, tender left periumbilical mass, Joints examination showed active synovitis involve meta-carbophalengial joints bilaterally, Knees $\&$ shoulders pain with passive and active movements. General Investigations were done (Table 1) suggestive of SLE and the patient abdominal CT showed rounded lumber mass with a double layer concluded intestinal intussusception.

The patient was seen by the surgical team, underwent laparotomy with bowel resection, the biopsy

examination report multiple fragments measuring $16 \mathrm{~cm}$ with attached binding $12.5 \mathrm{~cm}$ showed congested vascular channels and hypertrophied muscular fibers with no granulomas or malignancy saw, all these features consistent with a diagnosis of intussusception, no features of granuloma or neoplastic cells.

The patient was diagnosed with a case of systemic lupus erythematosus complicated with intussusception and the possibility of lupus nephritis. post-operatively the patient received prednisone $30 \mathrm{mg}$ with tapering Hydroxychloroquine $200 \mathrm{mg}$ per day Azathioprine $50 \mathrm{mg}$ twice per day Lisinopril $20 \mathrm{mg}$ per day, tonics Bone and gastric protection, although, the patient therapy showed good response yet, she is still on regular hemodialysis because renal biopsy done to the patient showed grade 4 lupus nephritis.

\section{Discussion}

SLE is a multisystem autoimmune disease in which deposition of immune complexes and pathogenic autoantibodies lead to a wide variety of symptoms (8). Abdominal pain in SLE patients can occur for multiple reasons including severe lupus vasculitis presenting as gastrointestinal perforation or mesenteric thrombosis, acute pancreatitis, cholecystitis, lupus mesenteric vasculitis and hepatitis (9). Intussusception is a condition in which the proximal segment of the intestine telescoping into the distal part of it (10). Although, intussusception I uncommon in adults and more likely to occur during childhood but adult have an underlying pathology in approximately $90 \%$ of cases. An intussusception in the small intestine mainly due to secondary causes, either due to extraluminal or intraluminal lesions ( Meckel's diverticulum, lymphoma, lipoma, postoperative, adhesions and metastases), however, intussusception in the large bowel is mostly due to malignant aetiology (11). The surgical specimen can shows macroscopically aspects of intussusception in the intestine (Figure 1) as well as the microscopically histopathology features (Figure 2)

At usually computer tomography (CT) Scan for the abdomen can identify the potential cause. The presence of bowel with bowel configuration with or without the existence of adipose tissue and mesenteric vessels is pathognomonic for intussusception (5).

The pathogenesis of the intussusception in our patient related to SLE could be mostly the initial manifestation of the disease. Only seven cases of intussusception in Patients with SLE have been described in the literature. In four cases were secondary to lupus mesenteric vasculitis (LMV) (7), one of them lymphadenopathy was the main cause, one was related to Burkitt's lymphoma, one was secondary to changes in the peristalsis of oedematous small intestine $(4,7,12,13)$.

The first report has been published by Hermann (11), on a five years old child with known who had an intussusception as a complication to LMV. The exact pathology and mechanism of LMV causing intussusception is not fully understood (4). A potential explanation is that vascular necrosis occurred by diffuse vasculitis with partial devitalisation of the intestinal segment, this can cause the interruption of the normal neuromuscular function, with concomitant intussusception and possibility of venous infarction necessitating bowel resection (4).

Intussusception is a very serious complication carrying a high mortality rate (14), CT abdomen has a central role in early detection of LMV and delivers a precise imaging of the lesions. However, the detection of the underlying causative factor of the intussusception can be challenging due to differentiation from bowel wall edema may not be possible (figure 3). In our case the CT abdomen of the patient Showed bilaterally pleural effusion, moderate free peritoneal fluid collection (Ascites) and rounded lumber mass with double layers suggestive of intussusception. 
Another report described a known case of lupus with fungal infection with intussusception. It is not fully clear whether there is an association between these conditions (15). However, in our case intussusception occur few days after the patient present complaining from the rheumatological features.

In conclusion, a 40-year-old female presented with multiple joints pain, skin rash, chest pain, hair loss and intussusception, bowel resection was done and they found no evidence of malignancy, infection causes or mesenteric vasculitis. Therefore, it is more likely that the intussusception was a secondary cause to SLE in this patient.

\section{Acknowledgments}

The case was diagnosed and treated in Dr Elnour Mohammed Elagib rheumatology unite at Omdurman Military hospital, Khartoum, Sudan.

Ethical approval and consent to publish

Obtained

\section{Conflict of Interest}

Non

\section{Written consent from the patient}

Obtained

\section{Authors contributions}

All authors contributed equally

Funding

No fund have been received

\section{Authors' contribution}

All authors contributed equally

\section{Availability of data and materials}

All the data used in the study are available from the first and corresponding author on reasonable request.

\section{Reference}

1. Farshad S, Kanaan C, Savedchuk S, Karmo DS, Halalau A, Swami A. Systemic Lupus Erythematosus (SLE) with Acute Nephritis, Antineutrophil Cytoplasmic Antibody- (ANCA-) Associated Vasculitis, and Thrombotic Thrombocytopenic Purpura (TTP): A Rare Case Report with Literature Review. Case Rep Rheumatol. 2019;2019:8750306.

2. Chu YC, Hsu BB, Tseng KC. Lupus mesenteric vasculitis with GI and genitourinary tract involvement. Clin Gastroenterol Hepatol. 2014;12(8):e69-70; quiz e1-2, e3.

3. de Carvalho JF. Mesenteric vasculitis in a systemic lupus erythematosus patient with a low sledai: an uncommon presentation. Clinics (Sao Paulo). 2010;65(3):337-40.

4. Lin YJ, Chen PC, Chen HA. Mesenteric vasculitis causing ileocecal intussusception as the initial presentation of systemic lupus erythematosus: a case report. Clin Rheumatol. 2013;32 Suppl 1:S37-40.

5. Zhang J, Fang M, Wang Y, Mao J, Sun X. Intestinal pseudo-obstruction syndrome in systemic lupus erythematosus. Lupus. 2011;20(12):1324-8. 
6. Fukaya S, Yasuda S, Hashimoto T, Oku K, Kataoka H, Horita T, et al. Clinical features of haemophagocytic syndrome in patients with systemic autoimmune diseases: analysis of 30 cases. Rheumatology (Oxford). 2008;47(11):1686-91.

7. Glijn N, Korswagen LA, Lam-Tse WK. Systemic lupus erythematosus (SLE): an unusual cause of ileocolic intussusception. BMJ Case Rep. 2017;2017.

8. Albuquerque-Netto AF, Cavalcante EG, Sallum AM, Aikawa NE, Tannuri U, Silva CA. Mesenteric vasculitis in a juvenile systemic lupus erythematosus patient. Rev Bras Reumatol. 2013;53(2):219-22.

9. Malaviya AN, Sharma A, Agarwal D, Kapoor S, Garg S, Singh S, et al. Acute abdomen in SLE. Int J Rheum Dis. 2011;14(1):98-104.

10. Bergmann KR, Arroyo AC, Tessaro MO, Nielson J, Whitcomb V, Madhok M, et al. Diagnostic Accuracy of Point-of-Care Ultrasound for Intussusception: A Multicenter, Noninferiority Study of Paired Diagnostic Tests. Ann Emerg Med. 2021.

11. Hermann G. Intussusception secondary to mesenteric arteries. Complication of systemic lupus erythematosus in a 5-year-old child. JAMA. 1967;200(1):74-5.

12. Yagmur Y, Aldemir M, Buyukbayram H, Tacyildiz I. Multiple jejunal diverticulitis with perforation in a patient with systemic lupus erythematosus: report of a case. Surg Today. 2004;34(2):163-6.

13. Chang D-K, Yoo D-H, Kim T-H, Kim IS, Jun KY, Park MH, et al. Burkitt's lymphoma presenting as ileocaecal intussusception in systemic lupus erythematosus. Clinical rheumatology. 1999;18(3):253-6.

14. Yagmur Y, Gumus S. Burkitt's lymphoma causing intussusception in adults: report of two cases and review of the literature. Journal of Gastroenterology and Hepatology Research. 2015;4(7):1702-6.

15. Wei CC, Chen JH, Cheng HH. Systemic lupus erythematosus with intussusception: a case report. Zhonghua Yi Xue Za Zhi (Taipei). 1996;58(1):58-61.

16. Kaemmerer E, Tischendorf JJW, Steinau G, Wagner N, Gassler N. Ileocecal Intussusception with Histomorphological Features of Inflammatory Neuropathy in Adenovirus Infection. Gastroenterology Research and Practice. 2009;2009:579501.

17. Kim YH, Blake MA, Harisinghani MG, Archer-Arroyo K, Hahn PF, Pitman MB, et al. Adult intestinal intussusception: CT appearances and identification of a causative lead point. Radiographics. 2006;26(3):73344.

Table 1: Shows the lab results performed for the patient

\begin{tabular}{ll}
\hline Investigations & Results \\
\hline WBC & $6.8 \mathrm{cells} / \mathrm{mcl}$ \\
HB & $7.3 \mathrm{~g} / \mathrm{dl}$ \\
Platelet & $127 \mathrm{cells} / \mathrm{mcl}$ \\
ESR & $60 \mathrm{~mm} / \mathrm{hour}$ \\
Serum Urea & $32 \mathrm{mg} / \mathrm{dl}$ \\
S.Creatinine & $1.1 \mathrm{mg} / \mathrm{dl}$ \\
CRP & 20 \\
S.Albumin & $2.9 \mathrm{~g} / \mathrm{dl}$ \\
Total Protein & $5.9 \mathrm{G} / \mathrm{dl}$ \\
Total Bilirubin & $0.4 \mathrm{mg} / \mathrm{dl}$ \\
ALT & $33 \mathrm{U} / \mathrm{L}$ \\
AST & $27 \mathrm{U} / \mathrm{L}$ \\
ALP & $105 \mathrm{U} / \mathrm{L}(24-147 \mathrm{UL})$ \\
Urine General & Albumin, no casts
\end{tabular}




\begin{tabular}{ll}
\hline Investigations & Results \\
\hline & $7-9$ pus \\
Direct Coomb Test & $\begin{array}{l}\text { Negative } \\
\text { positive Antidouble stranded DNA Antihistone positive AntiRibosomal p protein positive Anti nu }\end{array}$ \\
\hline
\end{tabular}

Figure 1: Shows the morphological features of ileocecal surgical specimens with intussusception (16).

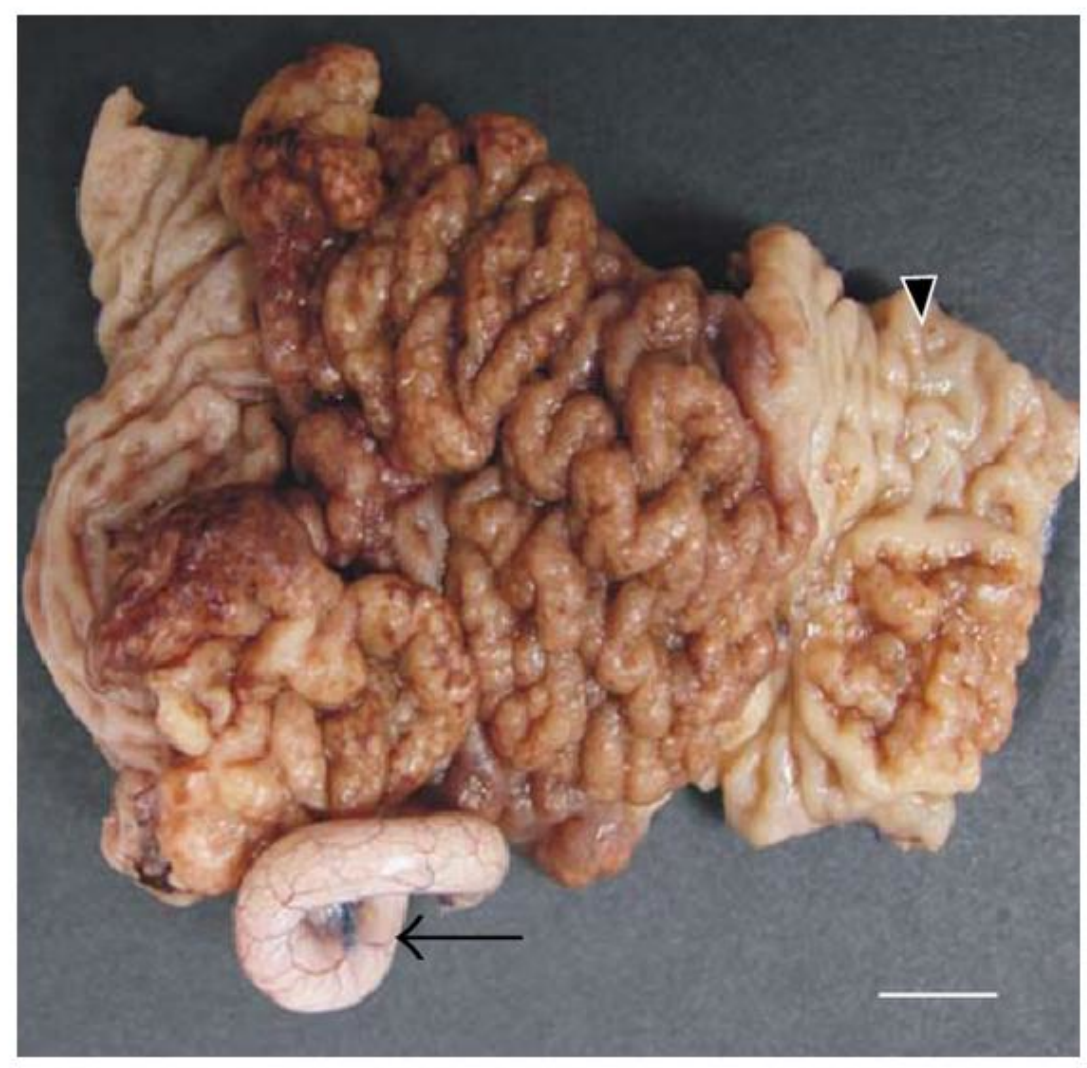

Figure 2: Tissue section of the terminal ileum demonstrates the plexus myentericus (dotted line) and several infiltrating lymphocytes and eosinophiles (arrowheads) (16). 


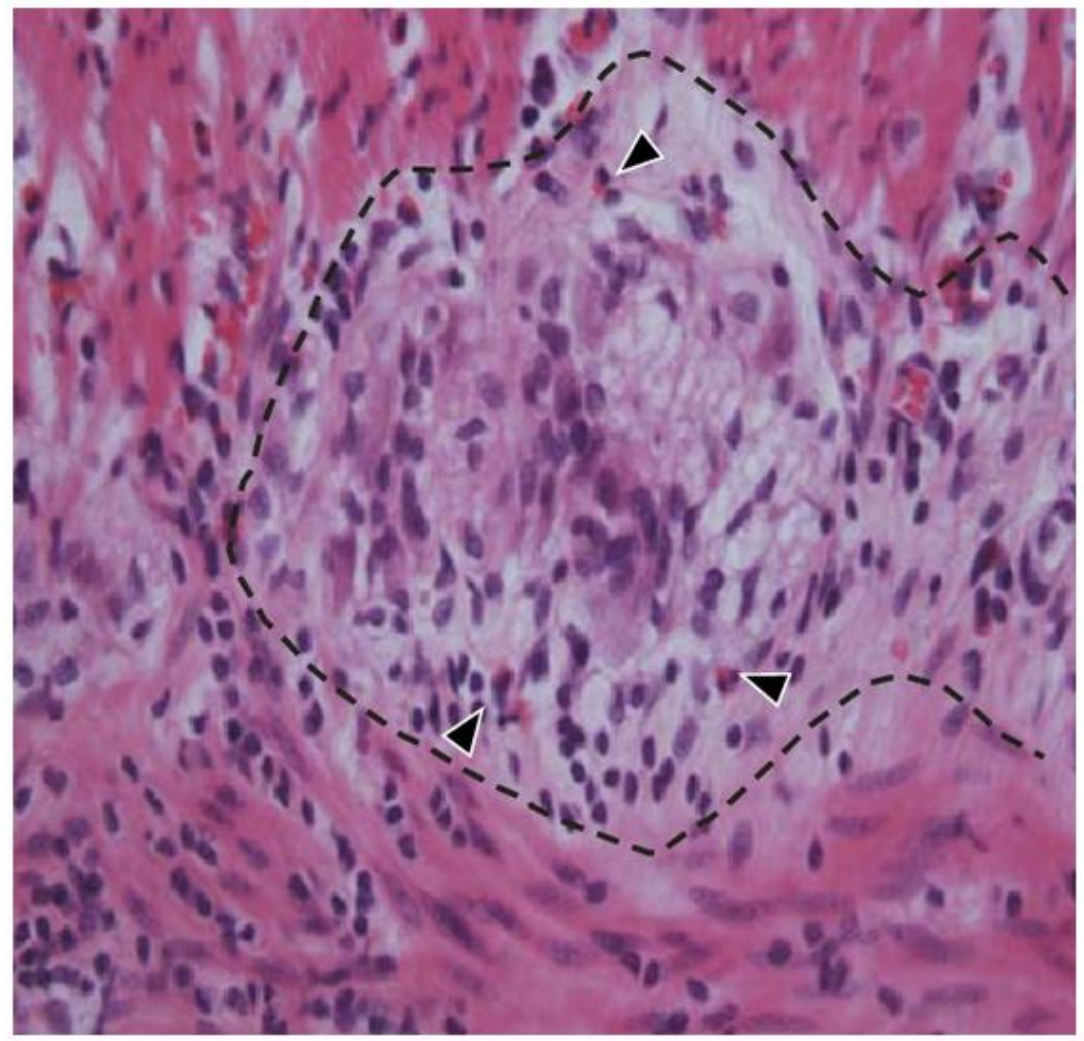

Figure (3): CT scans shows an intussusception in the abdomen, the mass is isoattenuating relative to bowel wall edema, making differentiation difficult (17) 


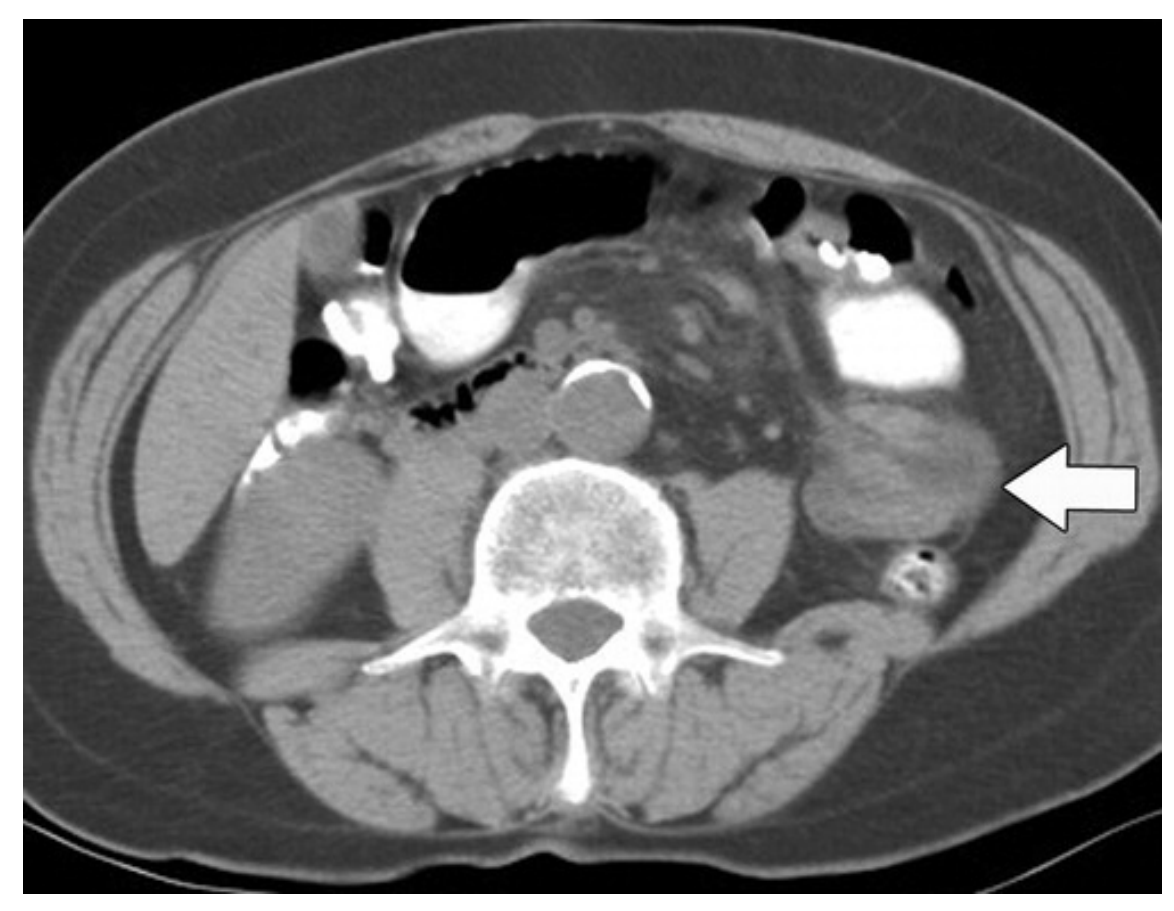

